\title{
Erratum
}

\section{Strain Driven Thermoelastic Instability Toward Brittle Fracture}

S. Boffi, C.E. Bottani, G. Caglioti, and P.M. Ossi

Z. Physik B 39, 135-141 (1980)

On page 141 Eq. (40) should read:

$\delta \varepsilon \cong \frac{\dot{\varepsilon}_{a}}{\omega} \overline{R(t)} \cos \omega t \cos k_{1} x$,

and Ref. 3 and 4 should be as follows:

3. Thomas, H.: In: Noise in physical systems. Wolf, D. (ed.), p. 278. Berlin, Heidelberg, New York: Springer 1978

4. Wallace, D.C.: Thermoelastic theory of stressed crystals and higher order elastic constants. In: Solid State Physics. Ehreinreich, H., Seitz, F., Turnbull, D. (eds.), Vol. 25, p. 301. New York: Academic Press 1970

S. Boffi

Istituto di Fisica Teorica

Università di Pavia

I-27100 Pavia

Italy

C.E. Bottani

G. Caglioti

P.M. Ossi

Istituto di Ingegneria Nucleare

CESNEF Politecnico di Milano

Via Ponzio, 34/3

I-20133 Milano

Italy 